\title{
QUALITY OF TRANSMISSION AWARE ROUTING IN AD HOC NETWORKS BASED ON CROSS-LAYER MODEL COMBINED WITH THE STATIC AGENT
}

\author{
LE HUU BINH ${ }^{1,2,3, a}$, VO THANH TU ${ }^{4}$, NGUYEN VAN TAM ${ }^{1,2, b}$ \\ ${ }^{1}$ Institute of Information Technology, Vietnam Academy of Science and Technology \\ ${ }^{2}$ Graduate University of Science and Technology, Vietnam Academy of Science and Tech. \\ ${ }^{3}$ Faculty of Information Technology, Hue Industrial College \\ abinh.lehuu@hueic.edu.vn; ${ }^{b}$ nvtam@ioit.ac.vn \\ ${ }^{4}$ Faculty of IT, College of Sciences, Hue University; vttu@hueuni.edu.vn
}

\begin{abstract}
The physical effects happening on the transmission routes in ad hoc networks influence the network performance seriously. These impacts decrease the quality of transmission, especially ad hoc networks with the wide area and high node density. This paper focused on investigating the routing techniques in ad hoc networks taking into account the quality of transmission. Thence, we proposed an improved routing algorithm of DSR based on the cross-layer model in combination with the static agent. The objective of the proposed algorithm is to improve the quality of the transmission signal, reduce the blocking probability of the data packets due to the unguaranteed quality of transmission.
\end{abstract}

Keywords. Ad hoc networks, cross-layer routing, QoT aware routing, sattic agent.

\section{INTRODUCTION}

In next generation network technologies, the wireless communications is one of the decisive solutions for the transmission technology of the telecommunications network in general and the computer network in particular. At the access layer, some models of wireless networks is being commonly used such as infrastructure networks, ad hoc networks. Among these models, ad hoc networks are becoming more and more widely used in many fields. There are three main types of ad hoc networks, which is wireless sensor network (WSN), mobile ad-hoc network (MANET) and wireless mesh network (WMN) [17]. The basic characteristics of ad hoc networks are the nodes which create peer to peer communication via wireless transmission medium, no control center for the data transmission in such networks. Nodes in ad hoc networks can operate as a client, a server as well as a router. Network topology changes frequently according to the random movement of nodes.

In order to increase the network performance of ad hoc networks, several published works have been reported recently. They focus on the control protocols for the transmission of data from source to destination, in which the routing protocols are the most studied. Most of published works related to routing protocols dedicate to improve the routing algorithms in order to decrease the probability of congestion, transmission delay, and increase the throughput of network $[5,7,9]$. The routing techniques in MANET taking into account the quality 
of transmission (QoT) have also recently attracted significant research interests from both academia and industry communities. The authors of [1] have modified the ad hoc on-demand distance vector (AODV) protocol in MANET using cross-layer model. The proposed algorithm uses three parameters of the quality link namely signal to noise ratio (SNR), delay and node lifetime to improve the network performance. Their method is to modify the RREP package by adding an extra fields in the packet structure to store the link cost value which is the summation of SNR, node lifetime and delay. Routing algorithm then chooses the route with the best link cost. Another published research has modified three routing protocols, AODV, Dynamic Source Routing (DSR) and Optimized Link State Routing (OLSR) to improve the performance of MANET [3, 8]. These protocols was modified by adding two fields in the route reply packet to store the metrics of SNR and received power (RP). The route with the best value of SNR or RP will be chosen for the data transmission.

Another method was used for the study of routing algorithms in MANET which takes into account the QoT is to use the routing metric. Specifically, it is constructing routing metrics, which contains the parameters of QoT. Then, the best path is selected based on this metrics. For this method, the authors of [12] proposed a routing metric namely Weighted Signal to noise ratio Average (WSA) for dynamic sequence distance vector (DSDV) routing protocol. The WSA metric uses the SNR parameter provided by the physical layer through crosslayer model. Simulation results showed that, for the use of WSA metric, the performance of MANET is improved in terms of throughput, packet delivery ration and end-to-end delay. In addition to the methods described above, the method using fuzzy logic to study QoT aware routing algorithms in MANET has also been deployed. The authors of [14] proposed a routing protocol namely Efficient Routing Protocol under Noisy Environment (ERPN) using fuzzy logic. The ERPN protocol select the route for transmission of data packets based on the parameters of the environment noise and signal strength. Their validation shows that, the ERPN protocol increases throughput, delivery ratio, decreases link failure, lowers error rate.

Based on several published works as described above, we could conclude that, the QoT aware routing techniques have attracted significant research interests and have been deployed in several different methods. The main objectives of proposed routing algorithms are to reduce the blocking probability of data pakets due to unguaranteed quality of transmission, as well as increase the network performance. In [11], we investigated the impact of the noise on the performance of MANET using on-demand routing protocols. By the simulation method, we have demonstrated that, the noise happening on the transmission routes influence the network performance seriously. In this paper, we continue to develop this subject, but use other method and focus on ad hoc networks with wide area and high node density. We proposed a routing algorithm which takes into account the QoT in ad hoc networks namely Quality of Transmission using Agent in DSR (QTA-DSR) to improve the network performance. QTA-DSR algorithm uses the cross-layer model combined with static agent. The functions of static agent in each node are to collect and process the information of QoT, are presented in detail in section 3.2.. The parameters of QoT which are taken into consideration include SNR, signal power, end-to-end delay and residual energy of nodes.

The rest of this paper is organized as follows. Section 2. discusses the impacts of physical effects on the performance of ad hoc networks. Section 3. describes the routing techniques in ad hoc networks using cross-layer model and the proposed routing algorithm. Section 4. 
presents the simulation results and discussions. Finally, concluding remarks and prospects of future works are given in Section $5 .$.

\section{QOT PARAMETERS IN AD HOC NETWORKS}

In the case of ad hoc networks with wide area and high node density, the physical effects happening on the transmission routes influence the network performance seriously. In this section, we discuss the parameters of the physical effects that have the most influence on QoT of ad hoc networks, including the path loss of signal power, SNR, bit error rate (BER).

\subsection{Path loss}

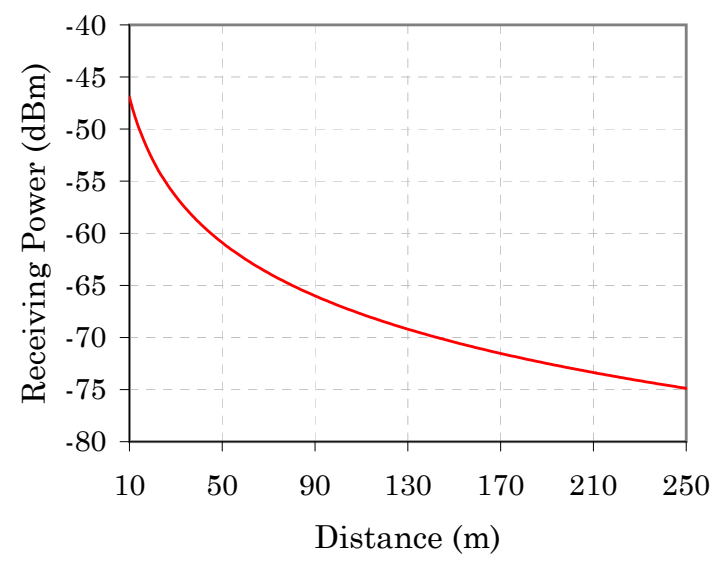

Figure 1. Receiving power decreases according to the transmission distance

In wireless propagation medium, if a signal transmitted through free space, the relation between the transmit and receive powers is given by [10]

$$
P_{R}=P_{T} G_{T} G_{R} \frac{\lambda^{2}}{(4 \pi d)^{2}}
$$

where $P_{T}$ and $P_{R}$ are the transmiting and receiving powers respectively, $G_{T}$ and $G_{R}$ are the transmiter and receiver antenna gains respectively, $\lambda$ is the wavelength of the carrier using in the modulation format, and $d$ is the distance between the transmiter and receiver. Equation (1) shows that the receiving signal power decreases according to the square of transmission distance, due to $P_{T}, G_{T}, G_{R}$ and $\lambda$ are constants. For example, considering the case of ad hoc network uses IEEE 801.11ac standard with the carrier of $5 \mathrm{GHz}$, assuming the transmiting power is $19.5 \mathrm{dBm}$, from equation (1), we obtain the curves of receiving power versus the transmission distance as shown in Figure 1. We can observe that, the receiving power decreases according to the transmission distance. This is the basis for setting the simulation parameters in the section 4.. For example, if the transmiting power is $19.5 \mathrm{dBm}$ and transmission range is $250 \mathrm{~m}$, receiver sensitivity must be $-75 \mathrm{dBm}$. 


\subsection{Signal to noise ratio $(\mathrm{SNR})$}

SNR is one of the important parameters to assess the quality of data channels in telecommunication networks, using both wired and wireless networks, which are defined as [14]

$$
S N R=10 \log _{10}\left(\frac{P_{s}}{P_{n}}\right)
$$

where $P_{s}$ and $P_{n}$ are the signal and noise powers respectively. For a data transmission channel, the higher SNR, the smaller BER and the better QoT. In ad hoc networks, for the cases that the data is transmitted through the many intermediate nodes, the noise power accumulated along the route increases, thus leading to the reduction of SNR according to (2). On the other hand, when the SNR decreases, the BER increases. Therefore, SNR constraint condition must be considered in the routing algorithms to ensure QoT. In order to evaluate the reduction of SNR in the abovementioned case, we consider a data transmission route from source to destination through the $m$ intermediate nodes ( $m-1$ hops) with the structure as shown in Figure 2. Then SNR at the receiver of the transmission channel is determined according to the following equation inverse [15]

$$
\frac{1}{S N R_{m}}=\sum_{i=1}^{m-1} \frac{1}{S N R_{h_{i}}}
$$

where $S N R_{m}$ is SNR value at destination node (node $m$ ), $S N R_{h_{i}}$ is SNR value of $i^{\text {th }}$ hop. To see more clearly SNR versus the number of hops that data packets transmitted in ad hoc networks, we consider the case of Mad hoc networks network with average SNR of each hop is $35 \mathrm{~dB}$, from equation (3), we obtain the curves of SNR and BER versus the number of hops as shown in Figure 3. From the curve in Figure 3(a) we thus can observe that, SNR decreases as data transmitted over multiple hops. If the data transmitted only one hop (without going through intermediate nodes), the SNR is $35 \mathrm{~dB}$. If it transmitted through two hops, SNR reduced to $32 \mathrm{~dB}$. This value reduced to $20.2 \mathrm{~dB}$ if data transmitted over 29 hops. Due to SNR reduction, BER increases as shown in Figure 3(b). Therefore, in order to ensure QoT in ad hoc networks, SNR constraint condition must be considered in the routing algorithms. This issue will be analyzed in the next section below.

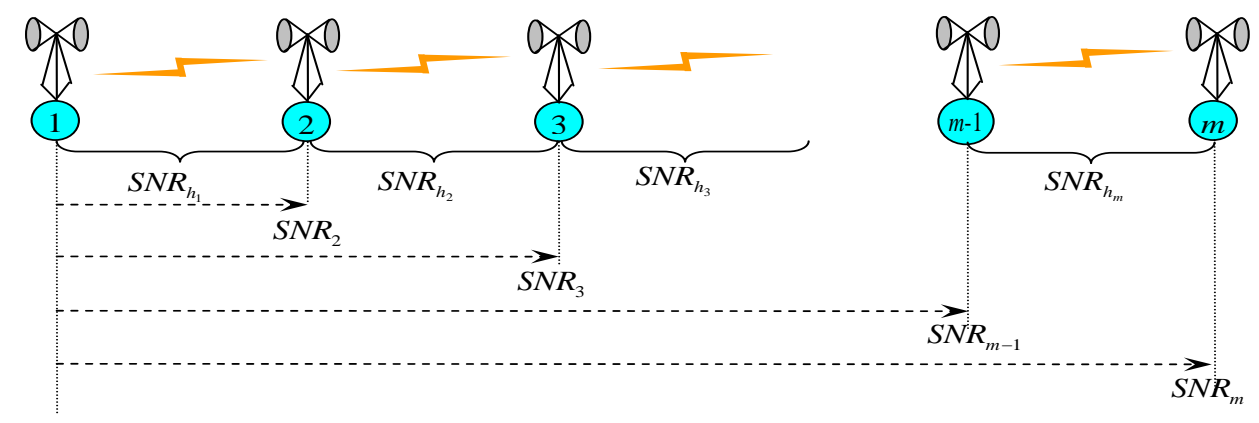

Figure 2. The structure of the data transmission route in ad hoc networks over multiple hops 


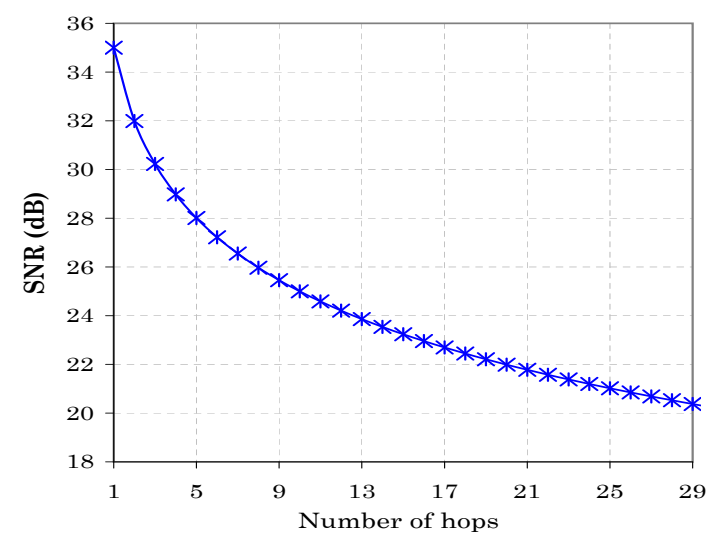

(a)

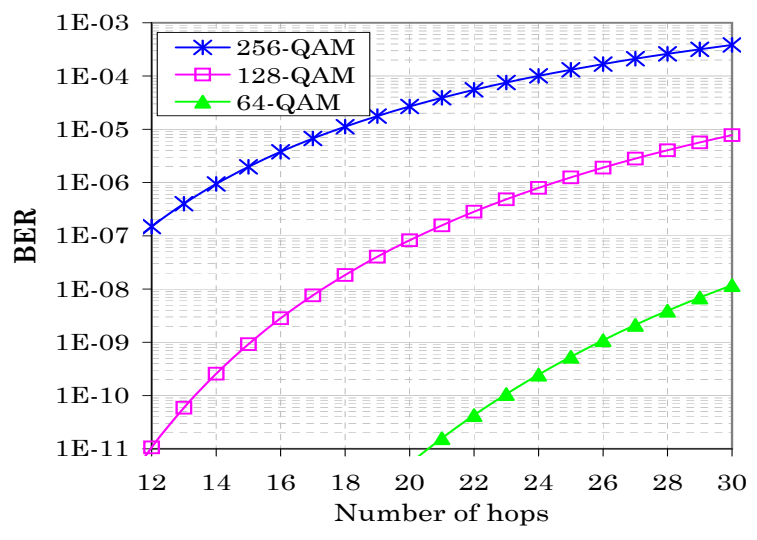

(b)

Figure 3. Characteristics of (a) SNR and (b) BER versus the number of hops

\subsection{End-to-end delay}

End-to-End delay is the summation of time taken by a data packet to travel from source to destination. For each hop from node $i$ to node $j$, delay consists of four components, namely processing delay $\left(\tau_{p}\right)$, queueing delay $\left(\tau_{q}\right)$, transmission delay $\left(\tau_{t}\right)$ and radio propagation delay $\left(\tau_{r}\right)$ [18]. Thus delay of each hop from node $i$ to node $j$ is determined by

$$
\tau_{i j}=\tau_{p}+\tau_{q}+\tau_{t}+\tau_{r}
$$

In the case of the processing delay and radio transmission delay are small enough to be able to ignore, $\tau_{i j}$ depends on two main components, $\tau_{t}$ and $\tau_{q} . \tau_{t}$ is determined based on channel bandwidth and data packet size, $\tau_{q}$ is based on the queue mechanism at the network nodes. In our model, $\mathrm{M} / \mathrm{M} / 1 / \mathrm{K}$ queuing is used at nodes, thus $\tau_{q}$ is determined by [19]

$$
\tau_{q}=\frac{\bar{N}}{\lambda\left(1-P_{K}\right)}+\frac{1}{\mu}
$$

where $\lambda$ and $\mu$ are the arrival rate and service rate (packets/s) of the link from $i$ to $j$, respectively. $\bar{N}$ is the average number of packets in the queue, $K$ is the capacity of queuing (packets) and $P_{K}$ is the probability of $K$ packets in queuing. $\bar{N}$ and $P_{K}$ are determined as follows [19]

$$
\begin{aligned}
\bar{N} & = \begin{cases}\frac{\rho}{1-\rho}-\frac{\rho\left(K \rho^{K}+1\right)}{1-\rho^{K+1}} & \text { if } \rho \neq 1 \\
\frac{K(K-1)}{2(K+1)} & \text { otherwise }\end{cases} \\
P_{K} & = \begin{cases}\frac{(1-\rho) \rho^{n}}{1-\rho^{K+1}} & \text { if } \rho \neq 1 \\
\frac{1}{K+1} & \text { otherwise }\end{cases}
\end{aligned}
$$

where $\rho=\lambda / \mu$ is the density of traffic offered to link from node $i$ to node $j$. In some optimal routing algorithms to ensure QoT in ad hoc networks, there are some cases the found route 
passing many intermediate nodes and hops, so end-to-end delay increases. In our proposed algorithm in section 3., the constraint condition of end-to-end delay is considered in order to ensure the delay within permissible limits.

\section{ROUTING ALGORITHM FOR ENSURING QoT IN AD HOC NETWORKS}

To improve the performance of ad hoc networks, we propose a routing algorithm which was modified from DSR algorithm, namely QTA-DSR (Quality of Transmission using Agent in DSR). The principle of the QTA-DSR algorithm is to integrate the parameters of QoT into the package RREQ including SNR, signal power, end-to-end delay and residual energy of nodes. This information is collected and processed by a static agent at each node. QTADSR algorithm selects a route with the best SNR in order to transmit the data from source to destination nodes, and simultaneously satisfy the constraint conditions of the end-to-end delay, residual energy of nodes and required SNR.

\subsection{Structure of RREQ and RREP packets}

Figures 4 and 5 show the structure of RREQ and RREP packets in QTA-DSR algorithm. RREQ packet of QTA-DSR algorithm is modified from that of DSR algorithm by adding two fields namely $S N R$ and $\tau$ in the packet structure (as shown in Figure 4) to store the SNR value and end-to-end delay. For RREP packet (Figure 5), we add a field namely SNR to store the SNR value from source to destination nodes of found routes. Based on this field, QTA-DSR algorithm a route with the best SNR for the data transmission.

\begin{tabular}{|c|c|c|}
\hline Option type & Opt Data Length & Identification \\
\hline \multicolumn{2}{|c|}{ Target Address } \\
\hline Address [1] \\
\hline Address [2] \\
\hline$\ldots$ \\
\hline Address [n]
\end{tabular}

(a)

\begin{tabular}{|c|c|c|}
\hline Option type & Opt Data Length & Identification \\
\hline Target Address \\
\hline Address [1] \\
\hline Address [2] \\
\hline A \\
\hline \multicolumn{3}{|c|}{ Address [n] } \\
\hline & SNR & $\tau$ \\
\hline
\end{tabular}

(b)

Figure 4. RREQ packet format of (a) DSR [6] and (b) QTA-DSR algorithms

\begin{tabular}{|c|c|c|c|}
\hline Opt type & Opt Data Len & Last Hop External & Reserved \\
\hline \multicolumn{4}{|c|}{ Address [1] } \\
\hline \multicolumn{4}{|c|}{ Address [2] } \\
\hline \multicolumn{4}{|c|}{$\ldots$} \\
\hline & & dress [n] & \\
\hline
\end{tabular}

(a)

\begin{tabular}{|c|c|c|}
\hline \begin{tabular}{|l|l|} 
Opt type & Opt Data Len \\
\end{tabular} & Last Hop External & Reserved \\
\hline \multicolumn{3}{|c|}{ Address [1] } \\
\hline \multicolumn{3}{|c|}{ Address [2] } \\
\hline \multicolumn{3}{|c|}{$\ldots$} \\
\hline \multicolumn{3}{|c|}{ Address [n] } \\
\hline & & SNR \\
\hline
\end{tabular}

(b)

Figure 5. RREP packet format of (a) DSR [6] and (b) QTA-DSR algorithms 


\subsection{Structure of node and principles of QTA-DSR algorithms}

In order to use the parameters of QoT as routing metric, the network layer must be able to directly access to the information of the physical layer. This can only be performed by using cross-layer model $[1,13,16]$. In our proposed model, the exchange of cross-layer information is performed by static agent with the structure as shown in Figure 6. The functions of static agent in each node are information collection, information processing and action decision. Considering an intermediate node $j$, assuming that node $j$ received RREQ packet from node $i$, static agent will read firstly the information of QoT from the source node $(s)$ to node $i$ contained in the package RREQ. These information include the SNR value from source node to node $i\left(S N R_{s i}\right)$ and the end-to-end delay from source node to node $i\left(\tau_{s i}\right)$. Static agent then reads the QoT sensor information of the hop from $i$ to $j$ nodes which include the SNR value from $i$ to $j$ nodes $\left(S N R_{i j}\right)$, the hop delay from $i$ to $j$ nodes $\left(\tau_{i j}\right)$ according to (4) and residual energy of node $j\left(E_{j}\right)$. Based on the QoT information that static agent collected, static agent will calculate to determine the parameters of QoT from the source node to the node $j$, including $S N R_{s j}$ and $\tau_{s j}$. On this basis, the constraint conditions of QoT can be determined by equation system:

$$
\left\{\begin{array}{l}
S N R_{s j}>S N R_{t h} \\
\tau_{s j}<\tau_{t h} \\
E_{j}>E_{t h}
\end{array}\right.
$$

where $S N R_{t h}$ is the required SNR threshold, $\tau_{t h}$ is the limit of the end-to-end delay and $E_{t h}$ is the required residual energy of node $j$.

If the constraint conditions of QoT satisfy (8), RREQ packet will continue to be processed. Else, the RREQ package will be deleted. In case of the routes that satisfy the constraint conditions of QoT can be found, QTA-DSR algorithms will selects route with the best SNR value to transmit the data based on the information of SNR in RREP packet.

\subsection{Mathematical modeling of the QTA-DSR algorithm}

According to the operation principle of the QTA-DSR algorithm as described in section 4.1., the found route has the best SNR, simultaneously it satisfies the constraint conditions of QoT and the end-to-end delay. Thus, if letting $X=\left\{x_{i j}\right\}$ as vector denoting the selected route, with the value of the elements $x_{i j}$ is determined by

$$
x_{i j}= \begin{cases}1 & \text { if link } i \rightarrow j \text { is selected } \\ 0 & \text { otherwise }\end{cases}
$$

To see more clearly the signification of vector $X$, we consider an ad hoc network model as shown in Figure 7, each wireless connection from node $i$ to node $j$ is assigned a variable $x_{i j}$. Assuming that node 1 requests a route to node 4 . If the values of vector $X$ obtained after running the routing algorithm as shown in Figure 7, the found route is $1 \rightarrow 2 \rightarrow 3 \rightarrow 4$.

Letting $N$ as the set of all nodes in ad hoc network, with above mechanism, the QTADSR algorithm is equivalent to solving the integer linear programming (ILP) problem of 


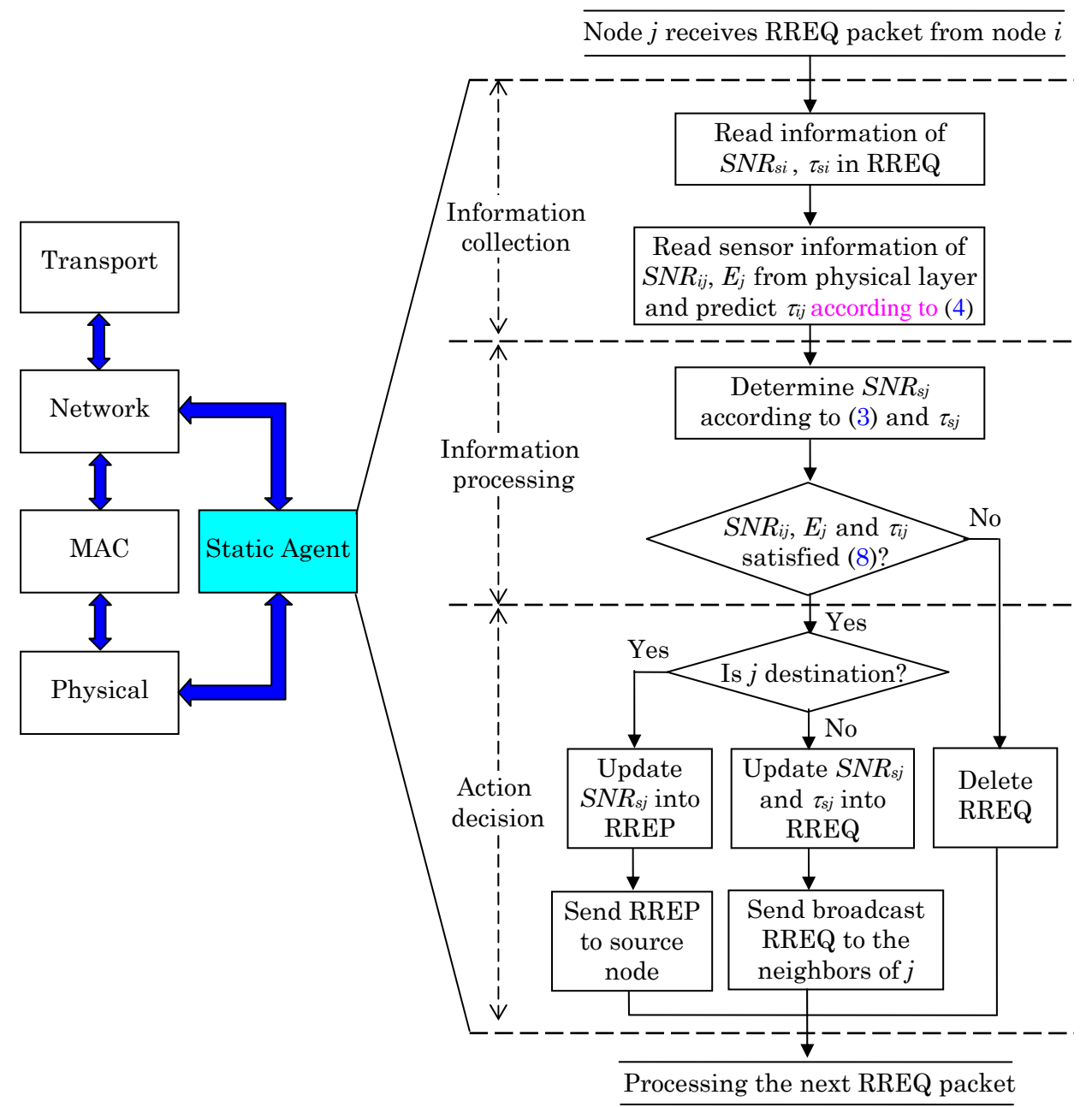

Figure 6. The structure of node in MANET according to cross-layer model using static agent

finding the vector $\left\{x_{i j}\right\}$. This can be achieved by the following ILP optimization:

$$
\text { Minimize } \sum_{(i, j) \in N}\left(\frac{1}{S N R_{i j}} x_{i j}\right)
$$

subject to the following constraints due to:

(i) The flow conservation constraints: For every node $j$ in network, if $j$ is the source $(s)$ or destination $(d)$ nodes, there are only the outgoing flow from $j$ or the incoming flow to $j$ respectively. Otherwise, for every node $j \notin\{s, d\}$, the incoming flow to $j$ must be equal to the outgoing. Thus the flow conservation constraints are determined by [4]

$$
\sum_{i \in N} x_{i j}-\sum_{k \in N} x_{j k}=\left\{\begin{aligned}
-1 & \text { If } j=s \\
1 & \text { If } j=d \\
0 & \text { otherwise. }
\end{aligned}\right.
$$

(ii) The end-to-end delay constraints: The end-to-end delay of data packet from source to destination nodes must be less than or equal to the limit of end-to-end delay $\left(\tau_{t h}\right)$, thus 


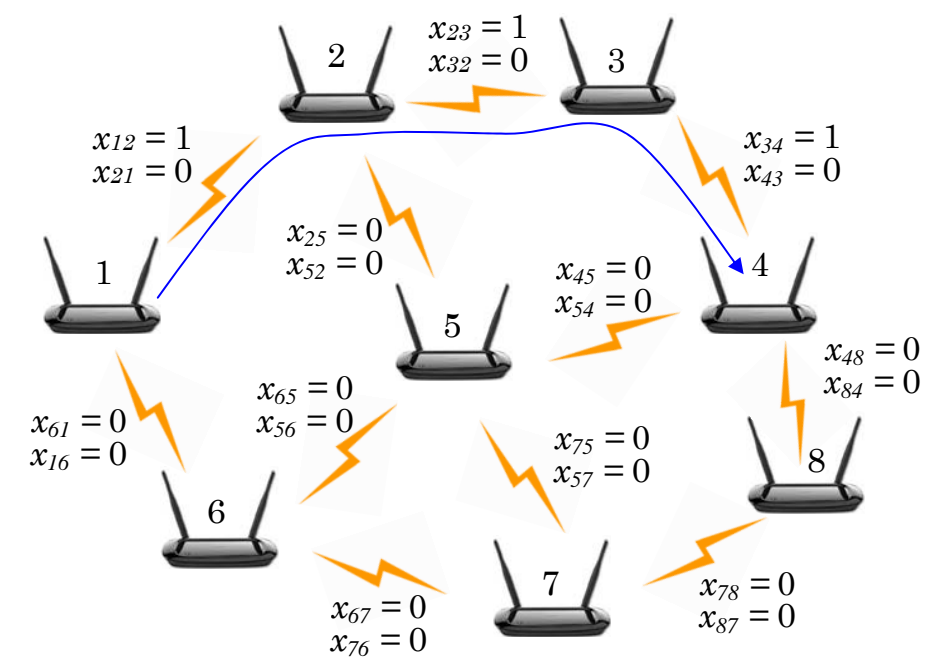

Figure \%. An example for the discovery of route in ad hoc network

we have

$$
\sum_{i, j \in N} \tau_{i j} x_{i j} \leq \tau_{t h}
$$

(iii) The SNR constraints: In order to QoT, SNR value at destination node must be greater than or equal to required SNR. Thus SNR constraints are determined by

$$
\sum_{i, j \in N} \frac{1}{S N R_{i j}} x_{i j} \leq \frac{1}{S N R_{t h}} .
$$

(iv) The residual energy of nodes constraints: To ensure that the data packets are not discarded at the intermediate nodes due to weak energy, the residual energy of each intermediate node along the route must be greater than the required energy. Thus we have

$$
\left(E_{j}-E_{t h}\right) x_{i j} \geq 0
$$

(v) The integer constraints: The values of vector $x_{i j}$ must be the binary and integer $(0$ or 1) according to (9). Thus we have

$$
\left(x_{i j}-1\right) x_{i j}=0 .
$$

By solving the ILP problem as described in (10) with the subject to the constraints from (11) to (15), we obtain the solution for $\left\{x_{i j}\right\}$, i.e. finding the route for data transmission that satisfies the constraint conditions of the quality of transmission.

\section{PERFORMANCE EVALUATION}

In this section, we evaluate the performance of QTA-DSR algorithm by the simulation method based on OMNeT++ [2] under operating system Linux Red Hat's Fedora Core 12. QTA-DSR algorithm is compared with DSR algorithm in terms of the BER, SNR, blocking 
probability of the data packet, and the end-to-end delay. Table 1 shows nine simulation scenarios for the cases of $10,15,20,25,30,35,40,45$ and 50 nodes. At the time of initialization, the position of the nodes was distributed uniformly in the simulated area. The pairs $(x, y)$ in Table 1 are the coordinates of the node. The number of source nodes is set

Table 1. The initial position of nodes and source nodes of the simulation scenarios

\begin{tabular}{|c|c|c|c|c|c|c|c|c|c|c|c|c|c|c|c|c|c|c|}
\hline \multirow[b]{2}{*}{ a } & \multicolumn{2}{|c|}{10 Nodes } & \multicolumn{2}{|c|}{15 Nodes } & \multicolumn{2}{|c|}{20 Nodes } & \multicolumn{2}{|c|}{25 Nodes } & \multicolumn{2}{|c|}{30 Nodes } & \multicolumn{2}{|c|}{35 Nodes } & \multicolumn{2}{|c|}{40 Nodes } & \multicolumn{2}{|c|}{45 Nodes } & \multicolumn{2}{|c|}{50 Nodes } \\
\hline & $\begin{array}{c}\text { Initial } \\
\text { Position }\end{array}$ & $\begin{array}{c}\text { Src } \\
\text { Node }\end{array}$ & $\begin{array}{c}\text { Initial } \\
\text { Position }\end{array}$ & $\begin{array}{c}\text { Src } \\
\text { Node }\end{array}$ & $\begin{array}{c}\text { Initial } \\
\text { Position }\end{array}$ & $\begin{array}{c}\text { Src } \\
\text { Node }\end{array}$ & $\begin{array}{c}\text { Initial } \\
\text { Position }\end{array}$ & $\begin{array}{c}\text { Src } \\
\text { Node }\end{array}$ & $\begin{array}{c}\text { Initial } \\
\text { Position }\end{array}$ & $\begin{array}{c}\text { Src } \\
\text { Node }\end{array}$ & $\begin{array}{c}\text { Initial } \\
\text { Position }\end{array}$ & $\begin{array}{c}\text { Src } \\
\text { Node }\end{array}$ & $\begin{array}{c}\text { Initial } \\
\text { Position }\end{array}$ & $\begin{array}{c}\text { Src } \\
\text { Node }\end{array}$ & $\begin{array}{c}\text { Initial } \\
\text { Position }\end{array}$ & $\begin{array}{c}\text { Src } \\
\text { Node }\end{array}$ & $\begin{array}{c}\text { Initial } \\
\text { Position }\end{array}$ & $\begin{array}{c}\text { Src } \\
\text { Node }\end{array}$ \\
\hline 1 & $(76,80)$ & $\checkmark$ & \begin{tabular}{|l|}
$(64,108)$ \\
\end{tabular} & & \begin{tabular}{|l|}
$(64,108)$ \\
\end{tabular} & $\checkmark$ & $(56,68)$ & & \begin{tabular}{|l|}
$(176,164)$ \\
\end{tabular} & $\checkmark$ & $(56,68)$ & & $(56,68)$ & $\checkmark$ & $(56,68)$ & & $(56,68)$ & \\
\hline 2 & $(44,216)$ & & $(488,168)$ & $\checkmark$ & $(488,168)$ & & $(344,408)$ & $\checkmark$ & $(920,320)$ & & $(920,320)$ & $\checkmark$ & $(920,320)$ & & $(920,320)$ & $\checkmark$ & $(920,320)$ & $\checkmark$ \\
\hline 3 & $(76,320)$ & & $(184,200)$ & & \begin{tabular}{|c|}
$(664,416)$ \\
\end{tabular} & & \begin{tabular}{|l|}
$(664,192)$ \\
\end{tabular} & & \begin{tabular}{|c|}
$(992,440)$ \\
\end{tabular} & & \begin{tabular}{|c|}
$(992,440)$ \\
\end{tabular} & & $(992,440)$ & & $(992,440)$ & & $(992,440)$ & \\
\hline 4 & $(290,30)$ & & \begin{tabular}{|l|}
$(264,304)$ \\
\end{tabular} & & \begin{tabular}{|l|}
$(640,120)$ \\
\end{tabular} & & \begin{tabular}{|l|}
$(560,184)$ \\
\end{tabular} & & $(560,168)$ & $\checkmark$ & $(952,40)$ & & $(952,40)$ & $\checkmark$ & $(952,40)$ & & $(952,40)$ & $\checkmark$ \\
\hline 5 & $(172,140)$ & & $(392,248)$ & $\checkmark$ & \begin{tabular}{|l|}
$(544,488)$ \\
\end{tabular} & $\checkmark$ & \begin{tabular}{|c|}
$(752,528)$ \\
\end{tabular} & $\checkmark$ & $(752,528)$ & & \begin{tabular}{|l|}
$(752,528)$ \\
\end{tabular} & $\checkmark$ & $(752,528)$ & & $(752,528)$ & $\sqrt{ }$ & $(752,528)$ & \\
\hline 6 & $(212,288)$ & $\checkmark$ & $(232,480)$ & & \begin{tabular}{|l|}
$(232,480)$ \\
\end{tabular} & & \begin{tabular}{|l|}
$(400,528)$ \\
\end{tabular} & & $(400,528)$ & $\checkmark$ & \begin{tabular}{|c|}
$(400,528)$ \\
\end{tabular} & & $(400,528)$ & $\checkmark$ & $(400,528)$ & & $(400,528)$ & \\
\hline 7 & $(276,200)$ & & \begin{tabular}{|c|}
$(472,56)$ \\
\end{tabular} & $\checkmark$ & \begin{tabular}{|l|}
$(472,56)$ \\
\end{tabular} & $\checkmark$ & \begin{tabular}{|l|}
$(784,72)$ \\
\end{tabular} & & \begin{tabular}{|l|}
$(784,72)$ \\
\end{tabular} & & \begin{tabular}{|l|}
$(784,72)$ \\
\end{tabular} & $\checkmark$ & $(784,72)$ & & $(784,72)$ & $\checkmark$ & $(784,72)$ & $\checkmark$ \\
\hline 8 & $(428,68)$ & & $(288,160)$ & & \begin{tabular}{|l|}
$(288,160)$ \\
\end{tabular} & & \begin{tabular}{|l|}
$(448,200)$ \\
\end{tabular} & & $(448,200)$ & & $(448,200)$ & & $(448,200)$ & & $(448,200)$ & & $(448,200)$ & \\
\hline 9 & $(412,190)$ & $\checkmark$ & $(376,504)$ & & \begin{tabular}{|l|}
$(376,504)$ \\
\end{tabular} & & \begin{tabular}{|l|}
$(600,488)$ \\
\end{tabular} & & $(600,488)$ & $\checkmark$ & \begin{tabular}{|c|}
$(600,488)$ \\
\end{tabular} & & $(600,488)$ & $\checkmark$ & $(600,488)$ & & $(600,488)$ & $\checkmark$ \\
\hline 10 & $(332,331)$ & & \begin{tabular}{|l|}
$(200,64)$ \\
\end{tabular} & & \begin{tabular}{|l|}
$(200,64)$ \\
\end{tabular} & & \begin{tabular}{|l|}
$(200,64)$ \\
\end{tabular} & $\checkmark$ & $(200,64)$ & $\checkmark$ & \begin{tabular}{|l|}
$(200,64)$ \\
\end{tabular} & $\checkmark$ & $(200,64)$ & $\checkmark$ & $(200,64)$ & $\checkmark$ & $(200,64)$ & \\
\hline 11 & N/A & & \begin{tabular}{|l|}
$(344,56)$ \\
\end{tabular} & $\checkmark$ & \begin{tabular}{|l|}
$(344,56)$ \\
\end{tabular} & & \begin{tabular}{|l|}
$(376,56)$ \\
\end{tabular} & & \begin{tabular}{|l|}
$(376,56)$ \\
\end{tabular} & & $(376,56)$ & $\checkmark$ & $(376,56)$ & & $(376,56)$ & $\checkmark$ & $(376,56)$ & \\
\hline 12 & N/A & & \begin{tabular}{|l|}
$(72,264)$ \\
\end{tabular} & & \begin{tabular}{|l|}
$(72,264)$ \\
\end{tabular} & $\checkmark$ & \begin{tabular}{|l|}
$(136,240)$ \\
\end{tabular} & & $(136,240)$ & & \begin{tabular}{|l|}
$(136,240)$ \\
\end{tabular} & & $(136,240)$ & & $(136,240)$ & & $(136,240)$ & $\checkmark$ \\
\hline 13 & N/A & & $(144,384)$ & & \begin{tabular}{|l|}
$(144,384)$ \\
\end{tabular} & & \begin{tabular}{|l|}
$(112,512)$ \\
\end{tabular} & $\checkmark$ & $(112,512)$ & & $(112,512)$ & & $(112,512)$ & & $(112,512)$ & & $(112,512)$ & $\checkmark$ \\
\hline 14 & N/A & & $(520,344)$ & $\checkmark$ & \begin{tabular}{|l|}
$(728,264)$ \\
\end{tabular} & $\checkmark$ & \begin{tabular}{|l|}
$(376,288)$ \\
\end{tabular} & & $(832,432)$ & $\checkmark$ & $(1048,336)$ & & $(1144,520)$ & $\checkmark$ & $(1080,120)$ & & $(1184,560)$ & \\
\hline 15 & N/A & & $(376,384)$ & & \begin{tabular}{|l|}
$(616,240)$ \\
\end{tabular} & & \begin{tabular}{|l|}
$(48,184)$ \\
\end{tabular} & & \begin{tabular}{|l|}
$(960,176)$ \\
\end{tabular} & & $(848,424)$ & $\checkmark$ & $(1132,88)$ & & $(496,32)$ & $\checkmark$ & $(1132,88)$ & \\
\hline 16 & $\mathrm{~N} / \mathrm{A}$ & & N/A & & \begin{tabular}{|l|}
$(520,344)$ \\
\end{tabular} & & \begin{tabular}{|l|}
$(232,320)$ \\
\end{tabular} & & \begin{tabular}{|l|}
$(344,392)$ \\
\end{tabular} & & $(1048,216)$ & & $(1048,216)$ & & $(1048,216)$ & & $(1048,216)$ & \\
\hline 17 & N/A & & N/A & & \begin{tabular}{|l|}
$(376,384)$ \\
\end{tabular} & & \begin{tabular}{|l|}
$(640,392)$ \\
\end{tabular} & $\checkmark$ & $(896,520)$ & $\checkmark$ & \begin{tabular}{|l|}
$(896,520)$ \\
\end{tabular} & & $(896,520)$ & $\checkmark$ & \begin{tabular}{|l|}
$(896,520)$ \\
\end{tabular} & & $(896,520)$ & $\checkmark$ \\
\hline 18 & N/A & & N/A & & \begin{tabular}{|l|}
$(264,304)$ \\
\end{tabular} & $\checkmark$ & \begin{tabular}{|l|}
$(456,416)$ \\
\end{tabular} & $\checkmark$ & $(456,416)$ & & \begin{tabular}{|l|}
$(456,416)$ \\
\end{tabular} & $\checkmark$ & $(456,416)$ & & $(456,416)$ & $\checkmark$ & $(456,416)$ & \\
\hline 19 & N/A & & N/A & & \begin{tabular}{|l|}
$(392,248)$ \\
\end{tabular} & & \begin{tabular}{|l|}
$(744,344)$ \\
\end{tabular} & & $(744,344)$ & & \begin{tabular}{|l|}
$(744,344)$ \\
\end{tabular} & & $(744,344)$ & & $(744,344)$ & & $(744,344)$ & \\
\hline 20 & N/A & & N/A & & \begin{tabular}{|l|}
$(184,200)$ \\
\end{tabular} & $\checkmark$ & \begin{tabular}{|l|}
$(248,472)$ \\
\end{tabular} & & $(248,472)$ & & \begin{tabular}{|l|}
$(248,472)$ \\
\end{tabular} & & $(248,472)$ & & $(248,472)$ & & $(248,472)$ & $\checkmark$ \\
\hline 21 & N/A & & N/A & & N/A & & \begin{tabular}{|l|}
$(288,168)$ \\
\end{tabular} & & $(744,264)$ & $\checkmark$ & \begin{tabular}{|l|}
$(376,128)$ \\
\end{tabular} & & $(1104,360)$ & $\checkmark$ & $(568,584)$ & & $(1192,384)$ & \\
\hline 22 & N/A & & N/A & & N/A & & \begin{tabular}{|l|}
$(784,224)$ \\
\end{tabular} & $\checkmark$ & $(848,192)$ & & \begin{tabular}{|l|}
$(848,192)$ \\
\end{tabular} & $\checkmark$ & $(848,192)$ & & $(848,192)$ & $\sqrt{ }$ & $(848,192)$ & \\
\hline 23 & N/A & & N/A & & N/A & & \begin{tabular}{|l|}
$(576,72)$ \\
\end{tabular} & & \begin{tabular}{|l|}
$(576,72)$ \\
\end{tabular} & & \begin{tabular}{|l|}
$(576,72)$ \\
\end{tabular} & & $(576,72)$ & & $(576,72)$ & & $(576,72)$ & \\
\hline 24 & N/A & & N/A & & N/A & & \begin{tabular}{|l|}
$(568,312)$ \\
\end{tabular} & & $(568,312)$ & & \begin{tabular}{|l|}
$(568,312)$ \\
\end{tabular} & & $(568,312)$ & & $(568,312)$ & & $(568,312)$ & $\checkmark$ \\
\hline 25 & N/A & & N/A & & N/A & & \begin{tabular}{|l|}
$(56,384)$ \\
\end{tabular} & $\checkmark$ & \begin{tabular}{|l|}
$(56,384)$ \\
\end{tabular} & & \begin{tabular}{|l|}
$(56,384)$ \\
\end{tabular} & & $(56,384)$ & & $(56,384)$ & & $(56,384)$ & \\
\hline 26 & N/A & & N/A & & N/A & & N/A & & $(664,192)$ & $\checkmark$ & \begin{tabular}{|c|}
$(664,192)$ \\
\end{tabular} & & $(664,192)$ & $\checkmark$ & $(664,192)$ & & $(664,192)$ & \\
\hline 27 & N/A & & N/A & & N/A & & N/A & & \begin{tabular}{|l|}
$(376,288)$ \\
\end{tabular} & & \begin{tabular}{|l|}
$(376,288)$ \\
\end{tabular} & $\checkmark$ & $(376,288)$ & & $(376,288)$ & $\checkmark$ & $(376,288)$ & \\
\hline 28 & N/A & & N/A & & N/A & & N/A & & \begin{tabular}{|l|}
$(48,184)$ \\
\end{tabular} & & \begin{tabular}{|l|}
$(48,184)$ \\
\end{tabular} & & $(48,184)$ & & $(48,184)$ & & $(48,184)$ & \\
\hline 29 & N/A & & N/A & & N/A & & N/A & & \begin{tabular}{|l|}
$(232,320)$ \\
\end{tabular} & & \begin{tabular}{|l|}
$(232,320)$ \\
\end{tabular} & & $(232,320)$ & & $(232,320)$ & & $(232,320)$ & $\checkmark$ \\
\hline 30 & N/A & & N/A & & N/A & & N/A & & \begin{tabular}{|l|}
$(288,168)$ \\
\end{tabular} & $\checkmark$ & \begin{tabular}{|l|}
$(288,168)$ \\
\end{tabular} & & $(288,168)$ & $\checkmark$ & $(288,168)$ & & $(288,168)$ & \\
\hline 31 & $\mathrm{~N} / \mathrm{A}$ & & N/A & & N/A & & N/A & & N/A & & \begin{tabular}{|l|}
$(768,248)$ \\
\end{tabular} & $\checkmark$ & $(768,248)$ & & \begin{tabular}{|l|}
$(768,248)$ \\
\end{tabular} & $\checkmark$ & $(768,248)$ & \\
\hline 32 & N/A & & N/A & & N/A & & N/A & & N/A & & \begin{tabular}{|c|}
$(960,176)$ \\
\end{tabular} & & \begin{tabular}{|l|}
$(1048,40)$ \\
\end{tabular} & & $(1048,40)$ & & $(1048,40)$ & \\
\hline 33 & N/A & & N/A & & N/A & & N/A & & N/A & & \begin{tabular}{|c|}
$(664,400)$ \\
\end{tabular} & & $(664,400)$ & $\checkmark$ & $(664,400)$ & & $(664,400)$ & $\checkmark$ \\
\hline 34 & N/A & & N/A & & N/A & & N/A & & N/A & & \begin{tabular}{|l|}
$(344,400)$ \\
\end{tabular} & $\checkmark$ & $(344,400)$ & & $(344,400)$ & $\checkmark$ & $(344,400)$ & \\
\hline 35 & N/A & & N/A & & N/A & & N/A & & N/A & & \begin{tabular}{|c|}
$(552,152)$ \\
\end{tabular} & $\checkmark$ & $(1040,544)$ & & $(832,584)$ & & $(1040,544)$ & \\
\hline 36 & N/A & & N/A & & N/A & & N/A & & N/A & & N/A & & $(848,424)$ & & $(848,424)$ & & $(848,424)$ & $\checkmark$ \\
\hline 37 & N/A & & N/A & & N/A & & N/A & & N/A & & N/A & & $(1048,336)$ & & $(1048,336)$ & & $(1048,336)$ & \\
\hline 38 & N/A & & N/A & & N/A & & N/A & & N/A & & N/A & & $(960,176)$ & & \begin{tabular}{|c|}
$(960,176)$ \\
\end{tabular} & & $(960,176)$ & \\
\hline 39 & N/A & & N/A & & N/A & & N/A & & N/A & & N/A & & $(552,152)$ & $\checkmark$ & $(552,152)$ & & $(552,152)$ & \\
\hline 40 & N/A & & N/A & & N/A & & N/A & & N/A & & N/A & & $(376,128)$ & $\checkmark$ & $(376,128)$ & $\checkmark$ & $(376,128)$ & \\
\hline 41 & N/A & & N/A & & N/A & & N/A & & N/A & & N/A & & N/A & & $(168,416)$ & $\checkmark$ & $(168,416)$ & \\
\hline 42 & N/A & & N/A & & N/A & & N/A & & N/A & & N/A & & N/A & & $(160,144)$ & & $(160,144)$ & $\checkmark$ \\
\hline 43 & N/A & & N/A & & N/A & & N/A & & N/A & & N/A & & N/A & & $(896,16)$ & $\checkmark$ & $(896,16)$ & $\checkmark$ \\
\hline 44 & N/A & & N/A & & N/A & & N/A & & N/A & & N/A & & N/A & & $(680,16)$ & & $(680,16)$ & \\
\hline 45 & N/A & & N/A & & N/A & & N/A & & N/A & & N/A & & N/A & & $(256,584)$ & $\checkmark$ & $(256,584)$ & $\checkmark$ \\
\hline 46 & N/A & & N/A & & N/A & & N/A & & N/A & & N/A & & N/A & & N/A & & $(1192,216)$ & \\
\hline 47 & N/A & & N/A & & N/A & & N/A & & N/A & & N/A & & N/A & & N/A & & $(1080,464)$ & $\checkmark$ \\
\hline 48 & N/A & & N/A & & N/A & & N/A & & N/A & & N/A & & N/A & & N/A & & $(832,584)$ & \\
\hline 49 & N/A & & N/A & & N/A & & N/A & & N/A & & N/A & & N/A & & N/A & & $(568,584)$ & $\checkmark$ \\
\hline 50 & N/A & & N/A & & N/A & & N/A & & N/A & & N/A & & N/A & & N/A & & $(496,32)$ & \\
\hline
\end{tabular}


to $30 \%$ of the the number of nodes, the remaining nodes are the destination nodes. For example, for the scenarios of 30 nodes, there are 10 source nodes and 20 destination nodes. Source nodes are checked as shown in Table 1. The other assumptions are presented in table 2 , where, the parameters of transmiting power, receiver sensitivity, transmission range and band are set according to (1) (as analyzed in section 2.1.). Figure 8 shows a snapshot of the animation interface during the simulation performance, node 22 is sending RREQ packet to node 23 in order to discover the route for transmitting data packets to the destination.

Table 2. Simulation parameters

\begin{tabular}{|l|l|l|l|}
\hline Parameters & Value & Parameters & Value \\
\hline Network Size & $1000 \mathrm{~m} \times 1000 \mathrm{~m}$ & BER threshold & $10^{-6}$ \\
\hline Modulation technique & 256 -QAM & Minimum Required SNR & $23.5 \mathrm{~dB}$ \\
\hline MAC protocol & $802.11 \mathrm{ac}$ & Noise model & Thermal noise \\
\hline Data rate & $1730 \mathrm{Mbps}$ & Temperature & $300^{0} \mathrm{~K}$ \\
\hline Transmit Power & $19.5 \mathrm{dBm}$ & Mobility model & Random - Waypoint \\
\hline Receiver Sensitivity & $-75 \mathrm{dBm}$ & Speed of nodes & $5-20 \mathrm{~m} / \mathrm{s}$ \\
\hline Transmission Range & $250 \mathrm{~m}$ & Time simulation & 600 seconds \\
\hline
\end{tabular}

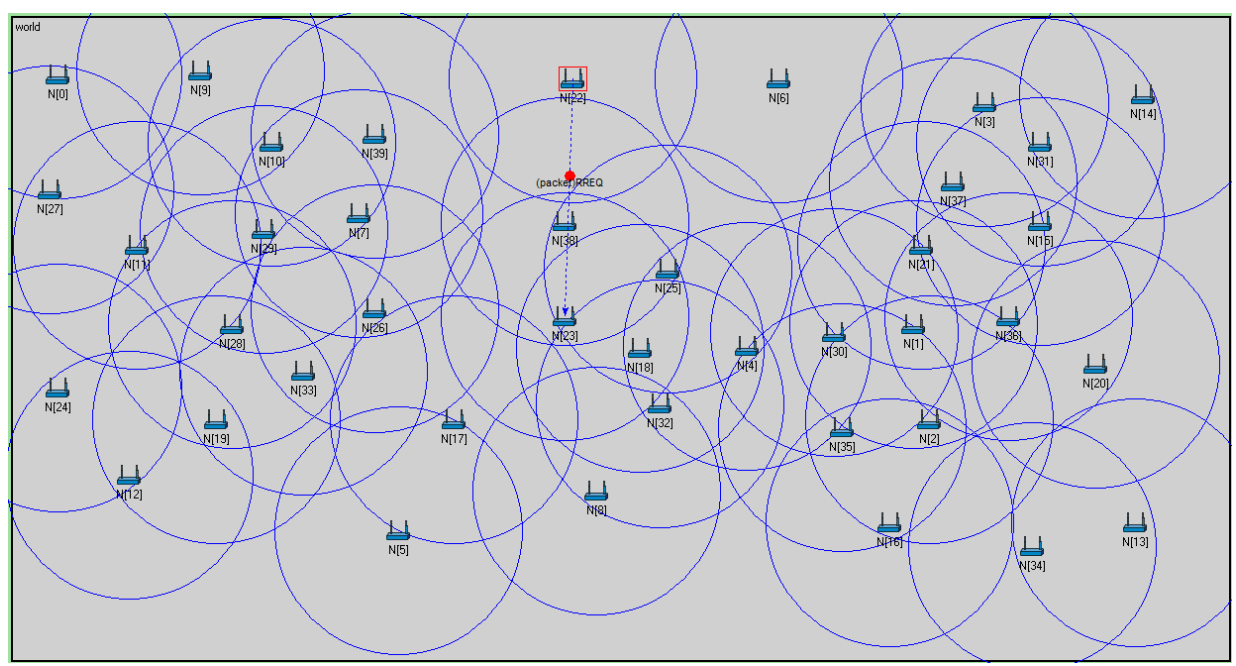

Figure 8. A snapshot of the animation interface during the simulation of QTA-DSR algorithm

\subsection{Evaluation of SNR}

The results obtained in Figure 9 shows the SNR versus the number of nodes. We can observe that, SNR decreases as the number of nodes increases. For DSR algorithm, SNR value is less than the required SNR $(23.5 \mathrm{~dB})$ in cases of the number of nodes is greater than 20 nodes. This causes the blocking packet probability increasing due to the unsatisfactory constraint conditions of QoT. 


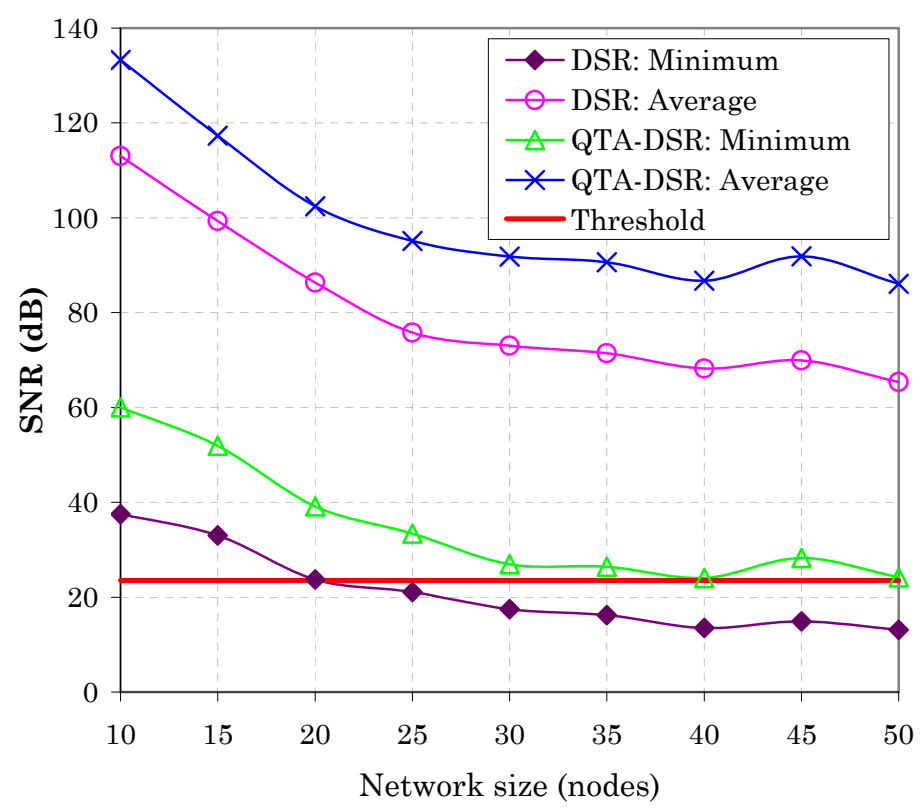

Figure 9. SNR versus the number of nodes for the cases that DSR and QTA-DSR

For QTA-DSR algorithm, SNR values improved significantly compared with DSR algorithm. In particular, the minimum SNR is always greater than the required SNR. Specifically, in case of the number of nodes is 25 , SNR value is $21.08 \mathrm{~dB}$ for the case that DSR algorithm is used. This value does not satisfy the required SNR threshold. For QTA-DSR algorithm, SNR value increases to $33.39 \mathrm{~dB}$, this value is greater than the required SNR threshold 9.89 dB. Thus QTA-DSR algorithm ensures the quality of transmission.

\subsection{Evaluation of Blocking Probability}

In this section, we discuss the blocking probability of data packets (BPD) in the overall network. In our context, BPD is given by

$$
B P D=\frac{N_{b}}{N_{g}}
$$

where $N_{g}$ and $N_{b}$ are the number of data packets are generated and the number of data packets are blocked in the overall network respectively. $N_{b}$ includes two components, blocking due to the congestion of the traffic load and blocking due to unsatisfactory constraint conditions of QoT.

As the SNR of QTA-DSR algorithm increases compared with DSR algorithm, BPD reduces in case of QTA-DSR algorithm is used. This is more clearly visible from Figure 10, where, we plot the BPD as a function of the traffic load. The curves in Figures 10a corresponding to the case of the number of nodes are 25. We can observe that, for the QTA-DSR algorithm, BPD is always less that of DSR algorithm. Considering the case of the traffic load is $50 \mathrm{Mbit} / \mathrm{s}$, BPD of DSR and QTA-DSR algorithms are $1.19 \times 10^{-2}$ and $1.1 \times 10^{-2}$ respectively. Thus BPD of QTA-DSR algorithm reduced to $41 \%$ compared with BPD of DSR algorithm. For the traffic load is high, such as $850 \mathrm{Mbit} / \mathrm{s}$, BPD of QTA-DSR algo- 
rithm reduced to $5.67 \%$ compared with $\mathrm{BPD}$ of DSR algorithm, from $1.46 \times 10^{-1}$ down to $1.37 \times 10^{-1}$.

Based on the results described above, we could conclude that, for the case of the number of nodes is 25, the difference in BPD of DSR and QTA-DSR algorithms is not too much. The main cause is that the reduction of SNR is not much in this case as analyzed in Figure 9. With the increasing number of nodes, the difference in BPD of two algorithms is very much. In the case where the number of nodes is 50, the curves of BPD versus Traffic load are shown in Figure 10b. We can observe that, for the QTA-DSR algorithm, the BPD reduced significantly compared with DSR algorithm. Specifically, for the traffic load of $50 \mathrm{Mbit} / \mathrm{s}$, BPD of DSR algorithm is $6.12 \times 10^{-2}$, meanwhile, BPD of QTA-DSR algorithm is only $2.82 \times 10^{-2}$. Compared with the DSR algorithm, BPD of QTA-DSR algorithm reduced to $53.9 \%$. Similarly, when the traffic load is greater than 50 to $850 \mathrm{Mbit} / \mathrm{s}$, BPD of QTADSR algorithm can reduce from $30 \%$ to $55 \%$. The cause of significant BPD reduction is the increasing SNR (as analyzed in Figure 9) which results in decreasing the number of blocked data packets due to the unsatisfactory constraint conditions of QoT.

From the results obtained in Figure 10, we can conclude that, the proposed algorithm effectively performs in case of the large network size. This is more clearly visible from Figure 11, where, we plot the BPD as a function of the network size in nodes. We can observe that, compared with DSR algorithm, the larger network size is, the more BPD reduced. Specifically, for the network size of 30 nodes, BPD of DSR and QTA-DSR algorithms are 0.058 and 0.047 respectively. Thus BPD of QTA-DSR algorithm reduced to $18.03 \%$ compared with DSR algorithm. When the network size of 50 nodes, BPD of QTA-DSR algorithm more significantly reduced, from 0.104 down to 0.068 , equivalent to $34.35 \%$. Thus QTA-DSR algorithm effectively performs in case of the network size is large.

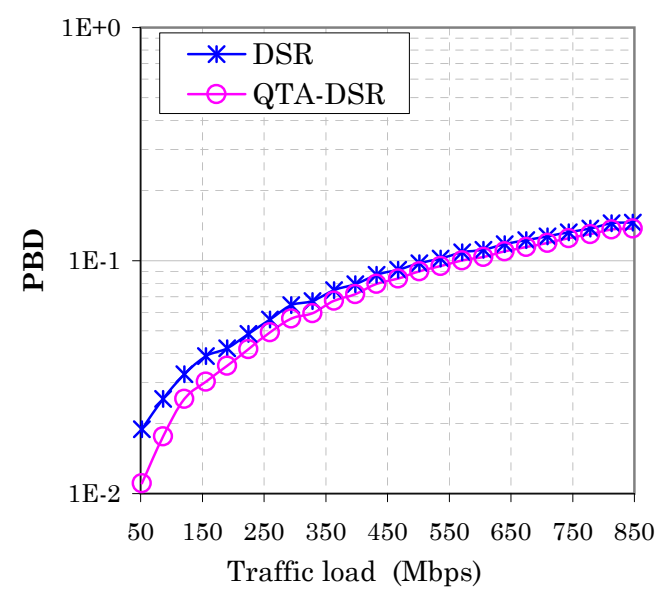

(a)

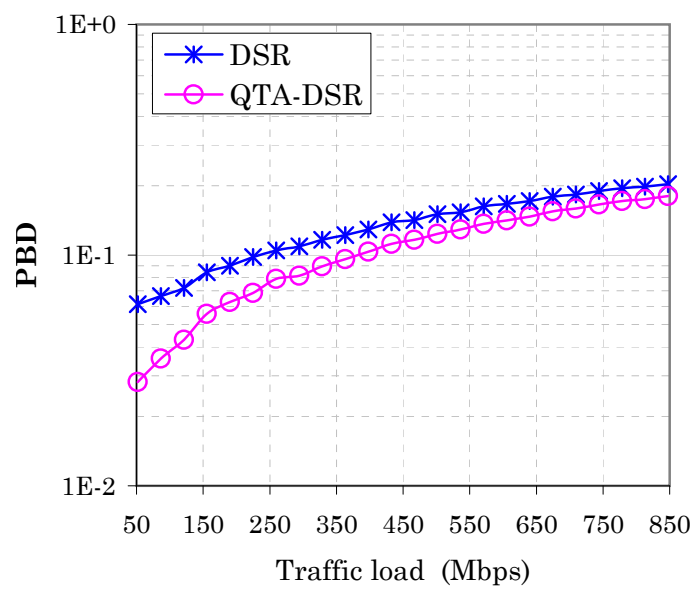

(b)

Figure 10. The comparision between BPD of DSR and that of QTA-DSR algorithms for the cases of (a) 25 nodes and (b) 50 nodes 


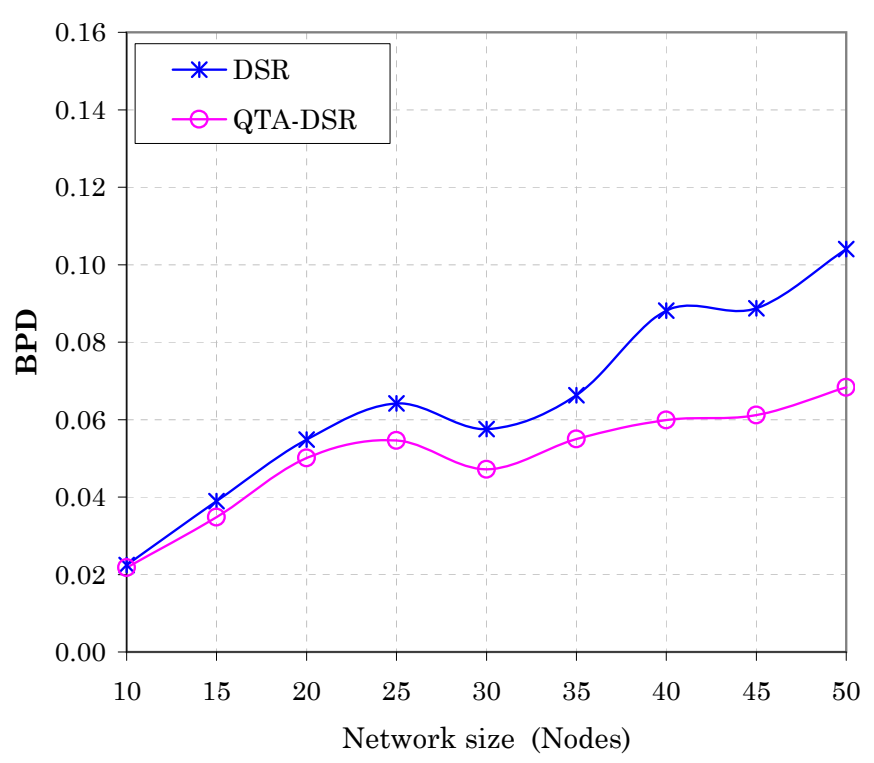

Figure 11. BPD versus Network size of DSR and QTA-DSR algorithms

\subsection{Evaluation of End-to-End Delay}

In this section, we discuss the average end-to-end delay of DSR and QTA-DSR algorithms. The simulation results are shown in Figure 12, where, we plot the average end-to-end delay as a function of the network size in nodes. We can observe that, the average end-to-end delay of DSR algorithm is close to that of QTA-DSR algorithm in the case of the small network size. For large network size, the average end-to-end delay of QTA-DSR algorithm is larger than that of DSR algorithm, however, the difference between the average end-to-end delay of QTA-DSR and that of DSR is negligible.

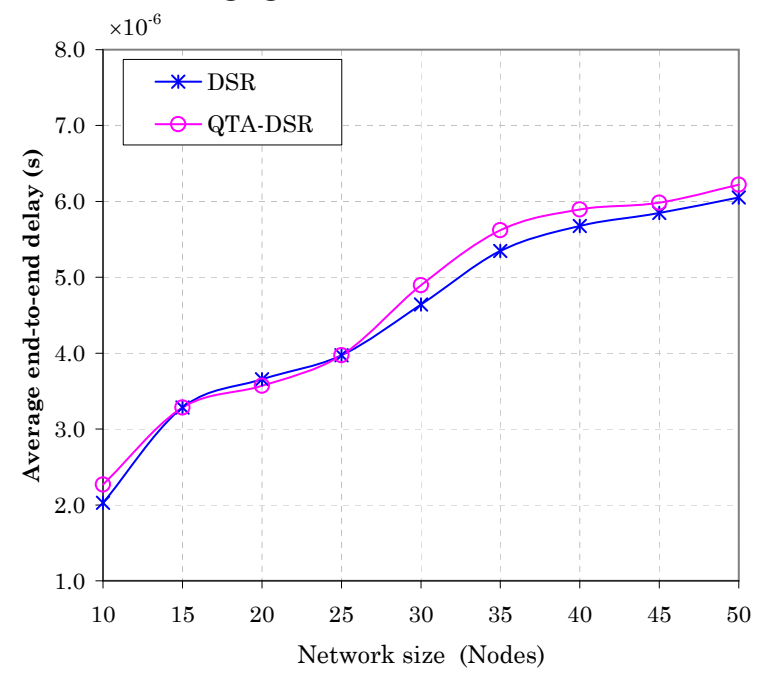

Figure 12. Average end-to-end delay versus Network size of DSR and QTA-DSR

From the results obtained in sections 4.1., 4.2. and 4.3., we can conclude that, the proposed algorithm (QTA-DSR) can select the route with the best QoT to data transmission, reduces BPD in the case of large network size, simultaneously, the end-to-end delay is within permissible limits. 


\section{CONCLUSION}

In order to improve the performance of ad hoc networks in the case of the wide area and high node density, it is essential to study the routing algorithm taking into account QoT. We presented in this paper the impact of the physical impairments on the performance of ad hoc networks. The physical impairments are considered including the loss of signal power, SNR, BER, end-to-end delay and residual energy of nodes. Thence, we proposed a routing algorithm that takes into account the constraint conditions of the physical impairments using cross-layer model in combination with static agent. The proposed algorithm is modified from DSR algorithm, namely QTA-DSR. The main objective of the QTA-DSR algorithm is to improve the QoT of ad hoc networks. By the simulation method, we have demonstrated that, the QTA-DSR algorithm can improve the SNR of the data transmission routes compared with DSR algorithm, reducing the blocking probability of data packets due to unsatisfactory constraint conditions of the quality of transmission.

We will report in the near future, the impact of the physical impairments with respect to the other routing protocols in ad hoc networks such as Ad hoc On-Demand Distance Vector (AODV) routing protocol, Destination-Sequenced Distance-Vector Routing (DSDV) protocol.

\section{ACKNOWLEDGMENT}

This research is funded by the Ministry of Education and Training under grant number B2016-DHH-21.

\section{REFERENCES}

[1] Aradhna Yadav et al., "Cross-Layer Approach For Communication in Manet", International Journal of Computer Science and Mobile Computing, Vol.4, No.3, 2015, pp. 285-292.

[2] Andras Varga, OMNeT++ Discrete Event Simulation System, Release 4.6, 2015. [Online]. Available: http://www. omnetpp.org

[3] F. Alnajjar and Y. Chen, "SNR/RP aware Routing algorithm: Cross-layer Design for MANETs", Int. J. of Wireless and Mobile Networks, Vol.1, No.2, 2009, pp.127-136.

[4] George B. Dantzig Mukund N. Thapa, Linear Programming, Springer-Verlag New York, LLC, 1997.

[5] Bakhsh, H. and Abdullah, M. "ARPM: Agent-based Routing Protocol For MANET", Int. J. Internet Protocol Technology, Vol. 3, No. 2, 2008, pp.136-146

[6] D. Johnson, Y. Hu and D. Maltz, "The Dynamic Source Routing Protocol (DSR) for Mobile Ad Hoc Networks for IPv4", RFC4728, [Online]. Available: http: //www.rfc-editor. org/rfc/rfc4728.txt

[7] Elis Kulla et al., "Investigation of AODV Throughput Considering RREQ, RREP and RERR Packets", $27^{\text {th }}$ International Conference on Advanced Information Networking and Applications, Barceona, Spain, March 2013, pp.169-174. 
[8] Fuad Alnajjar, "SNR/RP Aware Routing Model for MANETs", Journal of Selected Areas in Telecommunications (JSAT), 2011, pp. 40-48.

[9] Hong Li, Chu Dan, Wang Min, Li Shurong, "Mobile agent based Congestion Control AODV Routing Protocol", The $4^{\text {th }}$ International Conference on Wireless Communications, Networking and Mobile Computing, WiCOM 2008, pp.1-4.

[10] Kaveh P. and Prashant K., Principles Wireless Networks, Prenpice-Hall, Inc., 2002.

[11] Le Huu Binh, Vo Thanh Tu, "Evaluating the Impact of Noise with respect to Performance of MANET based on the on-demand Protocols, Proceedings of the $8^{\text {th }}$ National Conference on Fundamental and Applied Information Technology Research (FAIR'8), Ha Noi 9-10/07/2015, Publishing House Natural Science and Technology, 2015, pp. 119-126.

[12] Mohamed E. et al., "Weighted Signal-to-Noise Ratio Average Routing Metric for Dynamic Sequence Distance Vector Routing Protocol in Mobile Ad-Hoc Networks", IEEE $8^{\text {th }}$ Int. Colloquium on Signal Processing and its Applications (CSPA), 2012, pp. 329-334.

[13] M. M. Zoulikha and B. Amal, "Cross-layer approach among physical, MAC and routing layer in a shadowing environment", Ad-Hoc and Sensor Wireless Networks, Vol.21, No.1-2, 2014, pp.101-119.

[14] Supriya Srivastava, A. K. Daniel, "An Efficient Routing Protocol under Noisy Environment for Mobile Ad Hoc Networks using Fuzzy Logic", International Journal of Advanced Research in Artificial Intelligence, Vol. 2, No. 6, 2013, pp.34-39.

[15] Shafiullah K., Al-Sakib K. P., and Nabil A. A., Wireless Sensor Networks - Current Status and Future Trends, CRC Press, 2012.

[16] Vijayan R, Mareeswari V, Samyukta V, "Enhancing Energy Efficiency of Routing Protocol through Cross Layer Interactions in MANET", International Journal of Engineering and Technology (IJET), Vol. 5, No. 2, 2015, pp. 1147-1152.

[17] Jakub S. and Piotr Z., Multicast Routing in Wireless Mesh Networks, The Eigh ${ }^{\text {th }}$ Advanced International Conference on Telecommunications (AICT 2012), pp. 62-68.

[18] D. Bertsekas and R. Gallager, Data Networks, Second Edition, Prentice-Hall, 1992.

[19] Donald G., John F. Shortie, James M. T. and Carl M. H., Fundamentals of Queueing Theory, Fourth Edition, John Wiley \& Sons, Inc., Hoboken, New Jersey, 2008.

Received on October 18 - 2016

Revised on September 06 - 2017 